\title{
TOWARD A MATHEMATICAL THEORY OF AEROELASTICITY
}

\author{
A.V. Balakrishnan* \\ Flight Systems Research Center \\ UCLA \\ bal@ee.ucla.edu
}

Abstract This paper initiates a mathematical theory of aeroelasticity centered on the canonical problem of the flutter boundary - an instability endemic to aircraft that limits attainable speed in the subsonic regime. We develop a continuum mathematical model that exhibits the known flutter phenomena and yet is amenable to analysis - non-numeric theory. Thus we model the wing as a cantilever beam and limit the aerodynamics to irrotational, isentropic so that we work with the quasilinear Transonic Small Disturbance Equations with the attached flow and Kutta-Joukowsky boundary conditions. We can obtain a Volterra expansion for the solution showing in particular that the stability is determined by the linearized model consistent with the Hopf Bifurcation Theory, Specializing to linear aerodynamics, the time domain version of the aeroelastic problem is shown to be a convolution-evolution equation in a Hilbert space. The aeroelastic modes are shown to be the eigenvalues of the infinitesimal generator of a semigroup, which models the combined aerostructure state space dynamics. We are also able to define flutter boundary in terms of the "root locus" -- the modes as a function of the air speed $U$. We are able to track the dependence of the flutter boundary on the Mach number - a crucial problem in aeroelasticity - but many problems remain for Mach numbers close to one. The model and theory developed should open the way to Control Design for flutter boundary expansion.

\section{Introduction}

To a mathematician specializing in the problems of stability and control for partial differential equations, Aeroelasticity offers a fertile, if challenging, field of application. Currently, however, to a mathemati- 
cian - even an applied mathematician - Aeroelasticity (to paraphrase Richard E. Meyer, in Introduction to Mathematical Fluid Dynamics [1]) "appears to be built on a quicksand of assorted intuitions" - plus numerical approximations. This paper is a first halting step toward a "mathematical theory of Aeroelasticity."

The canonical problem of Aeroelasticity is flutter. It is an instability endemic to aircraft wings that occurs at high enough airspeed in subsonic flight and thus limits the attainable speed. The purpose of Control Design is to "expand" this "flutter boundary."

Control Design, however, requires a mathematical model that is simple enough for non-numeric analysis and yet displays the phenomena of interest - in this case flutter. In contrast almost all the extant work on this problem has been computational (see the review paper by Friedmann [2]). Computational techniques despite their success and universal use, require that numerical parameters be specified and thus cannot contribute to Control Design. The lack of a faithful enough mathematical model is undoubtedly one reason why all attempts at flutter control have failed so far. As we shall show, the kind of models needed require crucially recent advances in boundary control of partial differential equations. Even then many purely mathematical questions relating to the model are unanswered as yet.

We begin in Section 2 with the wing model, incorporating in addition a model for self-straining actuators. Section 3 is devoted to the aerodynamic model where we derive the TSD Equation from the Full Potential Equation clarifying the many assumptions made, and allowing for nonzero angle of attack. We linearize the TSD Equation and show it can be solved by the Possio Integral Equation, generalized to include nonzero angle of attack. We also develop a solution to the Linear Nonhomogeneous TSD Equation for zero initial and boundary conditions. Using these results we show how to construct a power series expansion - actually a Volterra kernel series expansion for the solution of the nonlinear TSD Equation. We are then able to obtain what is perhaps the most significant result - that the stability of the system is determined by the stability of the linear system - consistent with the Hopf Bifurcation Theory.

In Section 4 we go on to the abstract or time domain formulation of the flutter control problem. It turns out to be convolution-evolution equation in a Hilbert Space for the structure state - which is not quite the full state for which we used to go to a Banach Space formulation, enabling us to identify the aeroelastic modes as eigenvalues of the infinitesimal generator of the Banach Space semigroup. Of primary interest on the practical side is the calculation of these modes. This in turn 
leads to the "root locus" - the modes as a function of $U$ - and the definition of flutter speed. The dependence of the flutter speed on $M$ is an important unresolved issue here.

\section{The Wing Model}

The wing is modelled as a flexible structure - the flexibility is of course the key feature - as a "straight" uniform rectangular plate. Identifying the modes of the wing structure is one of the standard activities (vibration testing) in flight centers. The structure model must have the ability to conform to the first few measured modes at least. Following the model initiated by Goland [3] in 1954 we allow two degrees of freedom - plunge (displacement) and pitch (angle) about the elastic axis. Let

$$
x(s, t)=\left|\begin{array}{c}
h(s, t) \\
\theta(s, t)
\end{array}\right|, \quad 0 \leq t, \quad 0 \leq s \leq \ell,
$$

where $\ell$ is the wing span (one sided). Then the Goland model is:

$$
M_{s} \ddot{x}(s, t)+D_{s} \dot{x}(s, t)+K_{s} x(s, t)=\left|\begin{array}{c}
L(s, t) \\
M(s, t)
\end{array}\right|, \quad 0<s<\ell,
$$

where $K_{s}$ is the differential operator

$$
\begin{aligned}
K_{s} & =\left|\begin{array}{cc}
E I \frac{d^{4}}{d s^{4}} & 0 \\
0 & -G J \frac{d^{2}}{d s^{2}}
\end{array}\right| \\
M_{s} & =\left|\begin{array}{cc}
m & S \\
S & I_{\theta}
\end{array}\right| \\
\operatorname{det} M_{s} & >0 \\
D_{s} & =0
\end{aligned}
$$

and $L(s, t), M(s, t)$ denote the aerodynamic lift and moment. We are thus modelling the structure as a beam which would imply that the spread $2 b$ ("chord length") is "small" compared to the span $\ell$. Following Goland the beam is a cantilever clamped at the root $s=0$ and free at the tip $s=\ell$, so that we have the end conditions: at the root:

$$
\theta(0, t)=0 ; \quad h(0, t)=h^{\prime}(0, t)=0
$$

and at the tip:

$$
\theta^{\prime}(\ell, t)=0 ; \quad h^{\prime \prime}(\ell, t)=h^{\prime \prime \prime}(\ell, t)=0
$$


where the super primes denote derivative with respect to $s$ and the superdots denote time derivatives, in the usual notation.

We will need to change the tip conditions to:

$$
\left.\begin{array}{rl}
E I h^{\prime \prime}(\ell, t)+g_{h} \dot{h}^{\prime}(\ell, t) & =0 \\
G J \theta^{\prime}(\ell, t)+g_{\theta} \dot{\theta}(\ell, t) & =0
\end{array}\right\}
$$

if we wish to include a generally accepted model for self-straining actuators, with $g_{h}, g_{\theta} \geq 0$ being the gains.

\section{The Aerodynamic Model}

The aerodynamics is far the more complicated part. To comply with space limitation, the presentation will need to be quite compressed with minimal details of proofs.

To begin with, we shall assume the flow to be non-viscous. Next we will assume that it is isentropic and that the Perfect Gas Law applies. In this case, as shown in [4], the flow can be described by a velocity potential $\phi(x, y, z, t)$ which satisfies the so-called Full Potential Equation given by:

$$
\begin{aligned}
\frac{\partial^{2} \phi}{\partial t^{2}}+ & \frac{\partial}{\partial t}|\nabla \phi|^{2} \\
= & a_{\infty}^{2} \nabla^{2} \phi\left(1+\frac{\gamma-1}{a_{\infty}^{2}}\left(\frac{\left|q_{\infty}\right|^{2}}{2}-\frac{\partial \phi}{\partial t}-\frac{|\nabla \phi|^{2}}{2}\right)\right) \\
& \quad-\nabla \phi \cdot \nabla\left(\frac{1}{2}|\nabla \phi|^{2}\right), \quad-\infty<x, y, z<\infty, 0 \leq t
\end{aligned}
$$

where $q_{\infty}$ is the free stream (far-field) velocity and $a_{\infty}$ is the free stream (far-field) speed of sound, $\nabla$ denotes gradients in the usual notation, and

$$
M_{\infty}=\frac{\left|q_{\infty}\right|}{a_{\infty}}
$$

the far stream Mach number assumed $\leq 1$, and $\gamma$ is the ratio of specific heats.

This equation would appear to be complex but fortunately can be simplified since our primary concern is stability. Hence we go one level down to the Transonic Small Disturbance Equation - there are various versions [6], [10] but we shall follow Nixon [5] - see also [4]. Thus we assume that

$$
\varphi=\frac{\phi-\phi_{\infty}}{U}
$$

is "small" (see below for how it is used) where $\phi_{\infty}$ is the undisturbed or far stream potential:

$$
\phi_{\infty}=\left(x q_{1}+y q_{2}+z q_{3}\right) U
$$




$$
U^{2}=\left|q_{\infty}\right|^{2}, \quad q_{1}^{2}+q_{2}^{2}+q_{3}^{2}=1 .
$$

We have then the TSD Equation for $\varphi$ (see [7, equation 2.22]):

$$
\begin{aligned}
\frac{\partial^{2} \varphi}{\partial t^{2}}+2 U & \left(q_{1} \frac{\partial^{2} \varphi}{\partial x \partial t}+q_{2} \frac{\partial^{2} \varphi}{\partial y \partial t}+q_{3} \frac{\partial^{2} \varphi}{\partial z \partial t}\right) \\
= & a_{\infty}^{2}\left(1-M_{\infty}^{2} q_{1}^{2}-(1+\gamma) M_{\infty}^{2} q_{1} \frac{\partial \varphi}{\partial x}\right) \frac{\partial^{2} \varphi}{\partial x^{2}} \\
& +a_{\infty}^{2}\left(1-M_{\infty}^{2} q_{2}^{2}-(1+\gamma) M_{\infty}^{2} q_{2} \frac{\partial \varphi}{\partial y}\right) \frac{\partial^{2} \varphi}{\partial y^{2}} \\
& +a_{\infty}^{2}\left(1-M_{\infty}^{2} q_{3}^{2}-(1+\gamma) M_{\infty}^{2} q_{3} \frac{\partial \varphi}{\partial z}\right) \frac{\partial^{2} \varphi}{\partial z^{2}}
\end{aligned}
$$

Note that this is a quasi-linear equation with the right hand side neither elliptic nor hyperbolic, studied by Tricomi [6], Bers [7], Guderley [8], extensively, specialized to the stationary case.

\subsection{The Aeroelastic Problem}

Our interests are different in that we need to go beyond Transonic Aerodynamics to Transonic Aeroelasticity, as reflected in our preoccupation with the boundary conditions:

i) Flow Tangency Condition:

$$
\left.\frac{\partial \varphi}{\partial z}\right|_{z=0}=w_{a}(x, y, t):-b<s<b ; 0<y<\ell
$$

where $w_{a}(x, y, t)$ the "downwash" is the normal velocity of the structure. For our structure model of zero thickness, with $z(x, y, t)$ denoting the instantaneous displacement of the wing along the $z$-axis, we can calculate that:

$$
\begin{aligned}
w(x, y, t)= & \frac{D z(x, y, t)}{D t} \\
= & (-1)\left[\dot{h}(y, t)+(x-a b) \dot{\theta}(y, t)+U q_{1} \theta(y, t)\right], \\
& \quad-b<x<b, 0<y<\ell,|a|<1,
\end{aligned}
$$

where $x=a b$ locates the elastic axis of the wing in the $x y$ plane.

ii) Kutta-Joukowsky Conditions:

"Zero pressure jump off the wing and at the trailing edge" 
Now from [4] we have that pressure $p(x, y, z, t)$ can be expressed as

$$
p(x, y, z, t)=\frac{\rho_{\infty} a_{\infty}^{2}}{\gamma}\left(\frac{\rho}{\rho_{\infty}}\right)^{\gamma}
$$

where $\rho(x, y, z, t)$ is the density, and

$$
\rho=\rho_{\infty}\left[1+\frac{\gamma-1}{a_{\infty}^{2}}\left(\frac{1}{2} U^{2}-\psi(x, y, z, t)\right)\right]^{\frac{1}{\gamma-1}}
$$

where $\psi(x, y, z, t)$ is the acceleration potential

$$
\begin{aligned}
\psi & =\frac{\partial \phi}{\partial t}+\frac{1}{2}|\nabla \phi|^{2} \\
\psi_{\infty} & =\frac{1}{2} U^{2} .
\end{aligned}
$$

Now consistent with our small disturbance assumption,

$$
\frac{1}{2} U^{2}-\psi(x, y, z, t)
$$

can be approximated as

$$
\tilde{\psi}=\psi_{\infty}-\psi=U\left[-q_{\infty} \cdot \nabla \varphi-\frac{\partial \varphi}{\partial t}\right] .
$$

Further

$$
\left(1+\frac{\gamma-1}{a_{\infty}^{2}}\left(\psi_{\infty}-\psi\right)\right)^{\frac{1}{\gamma-1}}=\left(1+\frac{\gamma-1}{a_{\infty}^{2}} U\left(-q_{\infty} \cdot \nabla \varphi-\frac{\partial \varphi}{\partial t}\right)\right)^{\frac{1}{\gamma-1}}
$$

as in [4] can be approximated:

$$
=1+\frac{U}{a_{\infty}^{2}}\left[-q_{\infty} \cdot \nabla \varphi-\frac{\partial \varphi}{\partial t}\right] .
$$

Hence finally

$$
\left(\frac{\rho}{\rho_{\infty}}\right)^{\gamma}=\left(1+\frac{\gamma}{a_{\infty}^{2}} \tilde{\psi}\right)
$$

Hence the pressure jump

$$
\begin{aligned}
\delta p & =\left.(p(x, y, z, t)-p(x, y,-z, t))\right|_{z=0} \\
& =\rho_{\infty} \delta \tilde{\psi} .
\end{aligned}
$$


Hence we may express the Kutta-Joukowsky condition as:

$$
\left.\begin{array}{rl}
\delta \tilde{\psi} & =0, \quad|x|>b, 0<y<\ell \\
& =0, \quad x=b-, 0<y<\ell
\end{array}\right\}
$$

where

$$
\tilde{\psi}=\left(-q_{\infty} \cdot \nabla \varphi-\frac{\partial \varphi}{\partial t}\right) U .
$$

The lift $L(s, t)$ in $(1)$ is then given by

$$
L(y, t)=\rho_{\infty} \int_{-b}^{b} \delta \tilde{\psi} d x, \quad 0<y<\ell
$$

and so the moment $M(y, t)$ in (1) is given by

$$
M(y, t)=\rho_{\infty} \int_{-b}^{b}(x-a b) \delta \tilde{\psi} d x, \quad 0<y<\ell .
$$

We have thus completed our aeroelastic model, simplified to "small disturbance" theory. As Nixon notes in his review paper: The TSD (7) is the "minimum complexity equation that should be used for transonic flow prediction..." The first mathematical question is of course that of existence and uniqueness of solution for (7) subject to the stipulated boundary conditions. We may and do take the initial conditions to be zero, since we are interested only in the question of stability. Note that we have a "boundary-input" problem - the input being the normal velocity of the wing $w_{a}(x, y, t)$ and the "output" may be taken as $\tilde{\psi}(x, y, z, t)$. For arbitrary $w_{a}(x, y, t)$, we have thus a pure aerodynamic problem which we need to solve first. For the aeroelastic problem the function $w_{a}(x, y, t)$ is linear in the structure state:

$$
\begin{gathered}
\left|\begin{array}{c}
X(y, t) \\
\dot{X}(y, t)
\end{array}\right|=Z(y, t) \\
w_{a}(x, y, t)=\left[\ell_{1}, Z(y, t)\right]+x\left[\ell_{2}, Z(y, t)\right], \quad|x|<b .
\end{gathered}
$$

Heuristically then, by invoking physical realizability or Duhamel's principle we would have

$$
\begin{aligned}
L(\cdot, t) & =\int_{0}^{t} \mathcal{L}(Z(\cdot, \sigma), t-\sigma) d \sigma \\
M(\cdot, t) & =\int_{0}^{t} \mathcal{M}(Z(\cdot, \sigma), t-\sigma) d \sigma
\end{aligned}
$$


where $\mathcal{L}(\cdot, t), \mathcal{M}(\cdot, t)$ are nonlinear operators. This would make (1) a nonlinear "integro-differential" equation. We are primarily interested in the stability properties as a function of $U$ for fixed $M$, particularly in the transonic case for $M>0.8$.

\subsection{The Incompressible Case}

Before we proceed to the general problem there is one special limiting case - the "incompressible flow" case, corresponding to $M=0$ which is much the backbone of Aeroelasticity Theory. Thus we divide through by $a_{\infty}^{2}$ first in (7) and allow $M_{\infty} \rightarrow 0, a_{\infty} \rightarrow \infty$ but keeping

$$
U=a_{\infty} \cdot M_{\infty}
$$

finite. Then (7) (as well as in the Full Potential Equation (5)!) simplifies to

$$
\frac{\partial^{2} \varphi}{\partial x^{2}}+\frac{\partial^{2} \varphi}{\partial y^{2}}+\frac{\partial^{2} \varphi}{\partial z^{2}}=0, \quad-\infty<x, y, z<\infty
$$

so that the flow is incompressible and the field equation is stationary, but of course $U$ enters via the boundary conditions (9) and (10). Unfortunately this "3D problem for a finite wing" is still unsolved at this level of generality and we refer to [9] for a recent treatment.

\subsection{High-Aspect Ratio Wings: Typical Section Theory}

We now make a further simplifying assumption:

$$
\frac{\ell}{b} \approx \infty
$$

which is the mathematical equivalent of "high" aspect ratio wing. Or, formulated another way, we drop the dependence on $y$ so that we have a "typical section" theory. Thus (7) becomes

$$
\begin{aligned}
\frac{\partial^{2} \varphi}{\partial t^{2}}+2 U & \left(\cos \alpha \frac{\partial^{2} \varphi}{\partial x \partial t}+\sin \alpha \frac{\partial^{2} \varphi}{\partial z \partial t}\right) \\
= & a_{\infty}^{2}\left[1-M_{\infty}^{2} \cos ^{2} \alpha-(1+\gamma) M_{\infty}^{2} \cos \alpha \frac{\partial \varphi}{\partial x}\right] \frac{\partial^{2} \varphi}{\partial x^{2}} \\
& +a_{\infty}^{2}\left[1-M_{\infty}^{2} \sin ^{2} \alpha-(1+\gamma) M_{\infty}^{2} \sin \alpha \frac{\partial \varphi}{\partial z}\right] \frac{\partial^{2} \varphi}{\partial z^{2}}
\end{aligned}
$$


with the boundary conditions

$$
\begin{array}{rlrl}
\left.\frac{\partial \varphi}{\partial z}\right|_{z=0} & =w_{a}(x, s, t), & |x|<b, 0<x<\ell \\
& =(-1)[\dot{h}(s, t)+(x-a b) \dot{\theta}(s, t)+U \cos \alpha \theta(s, t)] .
\end{array}
$$

Note that as far as the aerodynamics is concerned, the span parameter $s$ is fixed. And the Kutta-Joukowsky conditions:

$$
\begin{aligned}
\delta \tilde{\psi} & =0, \quad|x|>b \\
& =0, \quad x=b-
\end{aligned}
$$

where

$$
\tilde{\psi}=U\left(\frac{\partial \varphi}{\partial t}+U \cos \alpha \frac{\partial \varphi}{\partial x}+U \sin \alpha \frac{\partial \varphi}{\partial z}\right) .
$$

There are as yet no general existence uniqueness results for this class of problems. This is typical for this area. Here we shall present a general solution technique.

\subsection{The Linear TSD Equation: Possio Integral Equation}

First however we need to consider the linear (or "linearized," as we shall show below) TSD which is obtained by eliminating the nonlinear or quasi-linear part -- that is setting

$$
(1+\gamma) M_{\infty}^{2}=0
$$

in (7a), but with the same boundary conditions, which would make the spatial part elliptic. Thus the linear TSD is

$$
\begin{aligned}
\frac{\partial^{2} \varphi}{\partial t^{2}}+ & 2 U\left(\cos \alpha \frac{\partial^{2} \varphi}{\partial x \partial t}+\sin \alpha \frac{\partial^{2} \varphi}{\partial z \partial t}\right) \\
& =a_{\infty}^{2}\left(\left(1-M_{\infty}^{2} \cos ^{2} \alpha\right) \frac{\partial^{2} \varphi}{\partial x^{2}}+\left(1-M_{\infty}^{2} \sin ^{2} \alpha\right) \frac{\partial^{2} \varphi}{\partial z^{2}}\right) .
\end{aligned}
$$

Details of the function space or "abstract" formulation of this problem for $\alpha=0$ are given in [11], and extended to the case $\alpha \neq 0$ in [4]. The technique is to go to the equivalent formulation as an integral equation called the Possio Integral Equation after the initiator Possio [12], after taking Laplace Transforms - (actually Fourier Transforms in the early 
work as in [12]). It is customary to do this in terms of the function

$$
\left.\begin{array}{rlrl}
A(x, t)=\frac{\delta P}{\rho_{\infty} U} & =\frac{-\delta \tilde{\psi}}{U}, & & |x|<b \\
& =0, & & |x|>b
\end{array}\right\} .
$$

Note that the Kutta condition requires

$$
A(b-, t)=0 .
$$

Because of the primary interest in stability, we work with Laplace Transforms. Thus let

$$
\hat{A}(x, \lambda)=\int_{0}^{\infty} e^{-\lambda t} A(x, t) d t, \quad \operatorname{Re} \lambda>\sigma_{a}
$$

and

$$
\hat{w}_{a}(x, \lambda)=\int_{0}^{\infty} e^{-\lambda t} w_{a}(x, t) d t .
$$

We have (the Possio Integral Equation valid for nonzero-angle-of-attack) normalizing $b$ to 1 and $\lambda$ to $k=\frac{\lambda b}{U}$ (see [4]).

$$
\hat{w}_{a}(x, \lambda)=\int_{-1}^{1} \hat{P}(x-\xi, \lambda) \hat{A}(\xi, \lambda) d \lambda, \quad|x|<1,
$$

where

$$
\begin{aligned}
& \int_{-\infty}^{\infty} \hat{P}(x, \lambda) e^{-i \omega x} d x \\
&=\frac{1}{k+i \omega \cos \alpha}\left(\frac{M^{2} k^{2}+2 M^{2} k i \omega \cos \alpha+\omega^{2}\left(1-M^{2} \cos ^{2} \alpha\right)}{\sqrt{M^{2} k^{2}+2 M^{2} k i \omega \cos \alpha+\omega^{2}\left(1-M^{2}\right)}}\right) \\
& \quad-\infty<\omega<\infty
\end{aligned}
$$

and $M_{\infty}$ is relaced by $M$, and

$$
\hat{w}_{a}(x, \lambda)=(-U)[k \hat{h}(s, \lambda)+(1-a k) \hat{\theta}(s, \lambda)+x k \hat{\theta}(s, \lambda)], \quad|x|<1 .
$$

For existence and uniqueness and abstract forulation of this problem see [11]. Here we shall simply assume this, so that in turn the linear TSD has a unique solution calculated via $\hat{A}(\cdot, \lambda)$ - as in [11]. For the aeroelastic problem the solution $\hat{A}(\cdot, \lambda)$ suffices.

Next we need to consider: 


\subsection{The Linear Nonhomogeneous TSD Equation}

In developing the solution to the nonlinear TSD Equation (7), we need to continue with the linear equation (7L) but now the nonhomogeneous case - nonzero right hand side. Thus we need to consider:

$$
\begin{gathered}
\frac{\partial^{2} \varphi}{\partial t^{2}}+2 U\left(\cos \alpha \frac{\partial^{2} \varphi}{\partial x \partial t}+\sin \alpha \frac{\partial^{2} \varphi}{\partial z \partial t}\right) \\
-a_{\infty}^{2}\left(1-M^{2} \cos ^{2} \alpha\right) \frac{\partial^{2} \varphi}{\partial x^{2}}-a_{\infty}^{2}\left(1-M^{2} \sin ^{2} \alpha\right) \frac{\partial^{2} \varphi}{\partial z^{2}} \\
=f(x, z, t), \quad-\infty<x, z<\infty ; 0<t
\end{gathered}
$$

with zero initial conditions:

$$
\begin{aligned}
& \varphi(x, z, 0)=0 \\
& \dot{\varphi}(x, z, 0)=0
\end{aligned}
$$

and zero boundary conditions

$$
\begin{aligned}
\left.\frac{\partial \varphi}{\partial z}\right|_{z=0} & =0, \quad|x|<1, \\
\delta \tilde{\psi} & =0, \quad-\infty<x<\infty
\end{aligned}
$$

and zero far-field conditions. In that case we can show that (7LNH) has a unique solution given in fact in terms of a Green's function:

$$
\begin{gathered}
\varphi(x, z, t)=\int_{0}^{t} \int_{-\infty}^{\infty} \int_{-\infty}^{\infty} g(x-\xi, y-\eta, t-\sigma) f(\xi, \eta, \sigma) d \xi d \eta d \sigma \\
-\infty<x, z<\infty
\end{gathered}
$$

for $f$ in the same space as $\varphi(\cdot)$. We skip the details.

Let us use the notation:

$$
\varphi=\mathcal{L} f
$$

where $\mathcal{L}$ is a linear bounded transformation.

Let us now return to the TSD (7). We shall outline a general technique of solution. The function space choices are described in [11]. We assume first that we have a solution which is analytic in the input $w_{a}(\cdot, \cdot)$, the initial conditions $\varphi$ and $\dot{\varphi}$ being zero at $t=0$. In other words we assume a solution $\varphi(\lambda)$ corresponding to the input $\lambda w_{a}(\cdot, \cdot)$ which is analytic in the complex variable $\lambda$. From the physical point of view, where we assume that the model also is, this goes without saying. Then $\varphi(\lambda)$ can 
be expanded in a power series in $\lambda$ about $\lambda=0$, valid for any $\lambda$ in the finite part of the plane:

$$
\varphi(\lambda)=\sum_{0}^{\infty} \lambda^{k} \varphi_{k}
$$

where

$$
\varphi(0)=0
$$

and

$$
\varphi_{k}=\left.\frac{\frac{d^{k}}{d \lambda^{k}} \varphi(\lambda)}{k !}\right|_{\lambda=0} .
$$

Now by (7), where $\varphi(\lambda)$ is more explicitly

$$
\varphi(x, z, t ; \lambda)
$$

we have

$$
\begin{gathered}
\frac{\partial^{2} \varphi(\lambda)}{\partial t^{2}}+2 U\left(\cos \alpha \frac{\partial^{2} \varphi(\lambda)}{\partial x \partial t}+\sin \alpha \frac{\partial^{2} \varphi(\lambda)}{\partial z \partial t}\right) \\
-a_{\infty}^{2}\left(1-M^{2} \cos ^{2} \alpha\right) \frac{\partial^{2} \varphi(\lambda)}{\partial x^{2}}-a_{\infty}^{2}\left(1-M^{2} \sin ^{2} \alpha\right) \frac{\partial^{2} \varphi(\lambda)}{\partial z^{2}} \\
=U^{2}(1+\gamma) \cos \alpha \frac{\partial \varphi(\lambda)}{\partial x} \frac{\partial^{2} \varphi(\lambda)}{\partial x^{2}} U^{2}(1+\gamma) \sin \alpha \frac{\partial \varphi(\lambda)}{\partial z} \frac{\partial^{2} \varphi(\lambda)}{\partial z^{2}}
\end{gathered}
$$

and

$$
\left.U \frac{\partial \varphi(\lambda)}{\partial z}\right|_{z=0}=\lambda w_{a}(t, x), \quad|x|<1 .
$$

Hence differentiating with respect to $\lambda$ in (24):

$$
\begin{aligned}
\left.U \frac{\partial}{\partial z} \frac{\partial \varphi(\lambda)}{\partial \lambda}\right|_{z=0} & =w_{a}(t, x), & |x|<1 \\
\left.U \frac{\partial}{\partial z} \frac{\partial^{k} \varphi(\lambda)}{\partial \lambda^{k}}\right|_{z=0}=0, & & |x|<1, k \geq 2
\end{aligned}
$$

and in $(23)$

$$
\begin{aligned}
& \frac{\partial^{2} \varphi_{k}}{\partial t^{2}}+2 U\left(\cos \alpha \frac{\partial^{2} \varphi_{k}}{\partial x \partial t}+\sin \alpha \frac{\partial^{2} \varphi_{k}}{\partial z \partial t}\right) \\
& -a_{\infty}^{2}\left(1-M^{2} \cos ^{2} \alpha\right) \frac{\partial^{2} \varphi_{k}}{\partial x^{2}}-a_{\infty}^{2}\left(1-M^{2} \sin ^{2} \alpha\right) \frac{\partial^{2} \varphi_{k}}{\partial z^{2}}
\end{aligned}
$$




$$
\begin{aligned}
=\left.k ! U^{2}(1+\gamma) \cos \alpha \frac{\partial^{k}}{\partial \lambda^{k}}\left[\frac{\partial \varphi(\lambda)}{\partial x} \frac{\partial^{2} \varphi(\lambda)}{\partial x^{2}}\right]\right|_{\lambda=0} \\
+\left.k ! U^{2}(1+\gamma) \sin \alpha \frac{\partial^{k}}{\partial \lambda^{k}}\left[\frac{\partial \varphi(\lambda)}{\partial z} \frac{\partial^{2} \varphi(\lambda)}{\partial z^{2}}\right]\right|_{\lambda=0}
\end{aligned}
$$

Hence, for $k=1$ we see that (27) reduces to the linear TSD Equation (7L) with the associated boundary conditions. The solution $\varphi_{1}$ is then uniquely determined via the corresponding nonzero-angle-of-attack Possio Integral Equation (17).

Next we see that $\varphi_{2}$ satisfies

$$
\begin{array}{r}
\frac{\partial^{2} \varphi_{2}}{\partial t^{2}}+2 U\left(\cos \alpha \frac{\partial^{2} \varphi_{2}}{\partial x \partial t}+\sin \alpha \frac{\partial^{2} \varphi_{2}}{\partial z \partial t}\right) \\
-a_{\infty}^{2}\left(1-M^{2} \cos ^{2} \alpha\right) \frac{\partial^{2} \varphi_{2}}{\partial x^{2}}-a_{\infty}^{2}\left(1-M^{2} \sin ^{2} \alpha\right) \frac{\partial^{2} \varphi_{2}}{\partial z^{2}} \\
=2 U^{2}(1+\gamma) \cos \alpha\left(2 \frac{\partial \varphi_{1}}{\partial x} \frac{\partial^{2} \varphi_{1}}{\partial x^{2}}\right) \\
+2 U^{2}(1+\gamma) \sin \alpha\left(2 \frac{\partial \varphi_{1}}{\partial z} \frac{\partial^{2} \varphi_{1}}{\partial z^{2}}\right)
\end{array}
$$

with

$$
\left.\frac{\partial \varphi_{1}}{\partial z}\right|_{z=0}=0, \quad|x|<1 .
$$

But this is the linear nonhomogeneous equation $(7 \mathrm{LNH})$ we have already treated. With $f_{2}$ denoting the right side of (28), we have that

$$
\varphi_{2}=\mathcal{L} f_{2} .
$$

More generally, with

$$
\begin{aligned}
f_{k}=k ! & \left.U^{2}(1+\gamma) \cos \alpha \frac{\partial^{k}}{\partial \lambda^{k}}\left(\frac{\partial \varphi(\lambda)}{\partial x} \frac{\partial^{2} \varphi(\lambda)}{\partial x^{2}}\right)\right|_{\lambda=0} \\
& +\left.k ! U^{2}(1+\gamma) \sin \alpha \frac{\partial^{k}}{\partial \lambda^{k}}\left(\frac{\partial \varphi(\lambda)}{\partial z} \frac{\partial^{2} \varphi(\lambda)}{\partial z^{2}}\right)\right|_{\lambda=0}
\end{aligned}
$$

we have that

$$
\varphi_{k}=\mathcal{L} f_{k}, \quad k \geq 2
$$


Hence we obtain:

$$
\varphi(\lambda)=\lambda \varphi_{1}+\sum_{2}^{\infty} \lambda^{k} \mathcal{L} f_{k}
$$

or, taking $\lambda=1$, the solution to (5) is given by

$$
\varphi=\varphi_{1}+\sum_{2}^{\infty} \mathcal{L} f_{k} .
$$

Our main interest in (31) is that of stability.

THEOREM 1 Suppose the linear solution $\varphi_{1}$ is stable. That is, denoting the dependence on $t$ by $\varphi_{1}(\cdot, t)$ suppose

$$
\varphi_{1}(\cdot, t) \rightarrow 0 \text { as } t \rightarrow \infty
$$

then so does $\varphi(\cdot, t)$. Also suppose $\varphi_{1}(\cdot, t)$ is periodic in $t$, then so is $\varphi(\cdot, t)$ with the same period.

REMARK We note that these statements are consistent with the central result of the more general Hopf Bifurcation Theory - as treated for example in [14]. In particular stability or instability is determined by the linearized equation.

Proof These results are easily deduced from the expansion (31). Thus if $\varphi_{1}$ is stable so is $\mathcal{L}\left(f_{k}\right)$ for each $k$. Similarly if $\varphi_{1}$ is periodic so is each $f_{k}$ and then also $\mathcal{L}\left(f_{k}\right)$ with the same period. QED.

REMARK We can show that the series (30) converges at a power series in $\lambda$ and that the function so defined satisfies (7).

Since our primary interest is in stability as a function of $U$ and by the Hopf Bifurcation Theory it is determined by the linear or linearized equation (7L) we shall now consider the linear problem in more detail, combining (1) and (7L). (It would appear that R. Triggiani in the paper presented at this conference (the 21 st IFIP TC-7 Conference) pursues a similar idea.)

\section{Time-Domain Formulation of Control Problem}

We now turn to the time-domain formulation of (1) with the aerodynamic lift and moment terms determined by the linearized equation (7L) (specializing to the typical section aerodynamics). We use the term 
"time-domain formulation" because in the aeroelastic literature, going back to the classic treatises [15], [16] only the Laplace Transform (or actually the Fourier Transform) theory is considered consistent with the primary interest in stability. Moreover abstract formulation as here is totally new.

We need first to calculate the lift $L(\cdot, t)$ and moment $M(\cdot, t)$, for which we use the solution to the Possion Equation (16). Here we take advantage of the speical form of $\hat{w}(\cdot, \lambda)$ in $(18)$ and introduce the functions

$$
\begin{aligned}
& f_{1}(x)=1, \quad|x|<1 \\
& f_{2}(x)=x, \quad|x|<1 .
\end{aligned}
$$

Let $\hat{A}_{i}(\cdot, \lambda)$ denote the solution to the Possio Equation:

$$
\int_{-1}^{1} \hat{P}(x-\xi) \hat{A}(\xi, \lambda) d \xi=f_{i}(x), \quad|x|<1
$$

and let

$$
\hat{w}_{i j}(M, k)=\int_{-1}^{1} f_{i}(x) \hat{A}_{j}(x, \lambda) d x
$$

as in [13]. Then

$$
\begin{aligned}
\hat{L}(s, \lambda)= & \int_{0}^{\infty} e^{-\lambda t} L(s, t) d t \\
= & -\rho b U^{2}\left[k \hat{w}_{11} \hat{h}(s, \lambda)+\left(k \hat{w}_{12}+(1-a k) \hat{w}_{11}\right) \hat{\theta}(s, \lambda)\right](32) \\
\hat{M}(s, \lambda)= & \int_{0}^{\infty} e^{-\lambda t} M(s, t) d t \\
= & -\rho b^{2} U^{2}\left[k\left(\hat{w}_{21}-\hat{w}_{11}\right) \hat{h}(s, \lambda)\right. \\
& \left.+\left(k \hat{w}_{22}+(1-a k) \hat{w}_{21}-a k \hat{w}_{12}-a(1-a k) \hat{w}_{11}\right) \hat{\theta}(s, \lambda)\right]
\end{aligned}
$$

Correspondingly, the inverse Laplace Transforms may be expressed

$$
\begin{aligned}
L(s, t) & =\int_{0}^{t} \ell(t-\sigma) y(s, \sigma) d \sigma \\
M(s, t) & =\int_{0}^{t} m(t-\sigma) y(s, \sigma) d \sigma
\end{aligned}
$$


where

$$
y(s, t)=\left|\begin{array}{c}
X(s, t) \\
\dot{X}(s, t)
\end{array}\right| .
$$

A minor complication in inverse Laplace Transforming is that we may have to allow for $\delta$ function and its derivative in $\ell(\cdot)$ and $m(\cdot)$, especially at $t=0$. This is illustrated by the case $M=0$ treated in [17]. Hence we shall write

$$
\begin{aligned}
& \begin{array}{c}
L(s, t) \\
M(s, t)
\end{array} \mid=M_{a} \ddot{x}(t)+D_{a} \dot{x}(t)+K_{a} x(t) \\
& +\left|\begin{array}{l}
\int_{0}^{t} \ell_{00}(t-\sigma) y(s, \sigma) d \sigma+\int_{0}^{t} \ell_{01}(t-\sigma) \dot{y}(s, \sigma) d \sigma \\
\int_{0}^{t} \ell_{10}(t-\sigma) y(s, \sigma) d \sigma+\int_{0}^{t} \ell_{11}(t-\sigma) \dot{y}(s, \sigma) d \sigma
\end{array}\right|
\end{aligned}
$$

(which only proves the efficiency of Laplace Transforms!) where we may assume (in the absence of proof here!) that

$$
M=M_{s}-M_{a}>0 \text { (nonsingular and nonnegative definite). }
$$

To proceed to the abstract formulation we need next to take care of the end conditions due to the possibility of self-training actuators. This means "including the boundary value as part of the state," initiated in [18], [19]. Thus let

$$
\begin{aligned}
\mathcal{H}_{b} & =L_{2} p[0, \ell] \times E^{1} \\
\mathcal{H}_{p} & =L_{2} p[0, \ell] \times E^{1} \\
\mathcal{H} & =\mathcal{H}_{b} \times \mathcal{H}_{p} .
\end{aligned}
$$

Define the linear operator

$$
A_{s}=\left|\begin{array}{cc}
A_{h} & 0 \\
0 & A_{\theta}
\end{array}\right|
$$

with domain and range in $\mathcal{H}$.

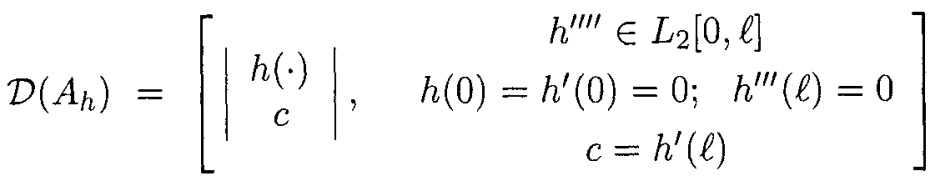

$$
\begin{aligned}
& A_{h}\left|\begin{array}{c}
h(\cdot) \\
h^{\prime}(\ell)
\end{array}\right|=\left|\begin{array}{c}
E \operatorname{EI}^{\prime \prime \prime \prime}(\cdot) \\
\operatorname{EIh}^{\prime \prime}(\ell)
\end{array}\right|
\end{aligned}
$$




$$
\begin{gathered}
\mathcal{D}\left(A_{\theta}\right)=\left[\begin{array}{c|c}
\theta(\cdot) & \theta^{\prime \prime} \in L_{2}[0, \ell] \\
c & \theta(0)=0 \\
c=\theta(\ell)
\end{array}\right] \\
A_{\theta}\left|\begin{array}{c}
\theta(\cdot) \\
\theta(\ell)
\end{array}\right|=\left|\begin{array}{c}
-G J \theta^{\prime \prime}(\cdot) \\
G J \theta^{\prime}(\ell)
\end{array}\right| .
\end{gathered}
$$

Thus defined, $A_{s}$ is self-adjoint, nonnegative definite with dense domain. Let $x \in \mathcal{D}\left(A_{s}\right)$. Then

$$
\left[A_{s} x, x\right]=E I \int_{0}^{\ell}\left|h^{\prime \prime}(s)\right|^{2} d s+G J \int_{0}^{\ell}\left|\theta^{\prime}(s)\right|^{2} d s
$$

where

$$
x=\left|\begin{array}{c}
h(\cdot) \\
h^{\prime}(\ell) \\
\theta(\cdot) \\
\theta(\ell)
\end{array}\right|
$$

and, in particular, we see that

$$
A_{s} x=0 \text { implies } x=0 .
$$

(There are no rigid-body modes.)

With $\sqrt{A_{s}}$ denoting the positive square root, we can verify that if $x \in \mathcal{D}\left(\sqrt{A_{s}}\right)$ we must have that

$$
x=\left|\begin{array}{c}
h(\cdot) \\
h^{\prime}(\ell) \\
\theta(\cdot) \\
\theta(\ell)
\end{array}\right| \text { is such that } \quad \begin{gathered}
h^{\prime \prime}(\cdot) \in L_{2}[0, \ell] \\
h(0)=L_{2}[0, \ell] \\
\theta(0)=0
\end{gathered} .
$$

Also, $\sqrt{A_{s}}$ has a bounded inverse.

Next we define the Hilbert space (energy space):

$$
\mathcal{H}_{E}=\mathcal{D}\left(\sqrt{A_{s}}\right) \times L_{2}[0, \ell]^{2}
$$

with inner product

$$
[Y, Z]_{E}=\left[\sqrt{A_{s}} x_{1}, \sqrt{A_{s}} x_{2}\right]+\left[M z_{1}, z_{2}\right]
$$

where

$$
Y=\left|\begin{array}{l}
x_{1} \\
z_{1}
\end{array}\right|, \quad Z=\left|\begin{array}{l}
x_{2} \\
z_{2}
\end{array}\right|
$$




$$
\begin{aligned}
& x_{1}=\left|\begin{array}{c}
h_{1} \\
h_{1}^{\prime}(\ell) \\
\theta_{1} \\
\theta_{1}(\ell)
\end{array}\right|, \quad z_{1}=\left|\begin{array}{c}
h_{2}(\cdot) \\
\theta_{2}(\cdot)
\end{array}\right| \\
& x_{2}=\left|\begin{array}{c}
h_{3} \\
h_{3}^{\prime}(\ell) \\
\theta_{3} \\
\theta_{3}(\ell)
\end{array}\right|, \quad z_{2}=\left|\begin{array}{c}
h_{4}(\cdot) \\
\theta_{4}(\cdot)
\end{array}\right|
\end{aligned}
$$

where

$$
M=\left(M_{s}-M_{a}\right)>0 .
$$

Define $\mathcal{A}_{s}$ with domain and range in $\mathcal{H}_{E}$ by:

$$
\begin{array}{r}
\mathcal{D}\left(\mathcal{A}_{s}\right)=\left[Y=\left|\begin{array}{l}
x_{1} \\
z_{1}
\end{array}\right|, x_{1}=\left|\begin{array}{c}
h_{1} \\
h_{1}^{\prime}(\ell) \\
\theta_{1} \\
\theta_{1}(\ell)
\end{array}\right|\right. \\
\text { and }\left|\begin{array}{c}
h_{2}\left(A_{s}\right), z_{1}=\left|\begin{array}{c}
h_{2}(\cdot) \\
\theta_{2}(\cdot)
\end{array}\right| \\
-E I \frac{h_{1}^{\prime \prime}(\ell)}{g_{h}} \\
\theta_{2} \\
-G J \frac{\theta_{1}^{\prime}(\ell)}{g_{\theta}}
\end{array}\right|
\end{array}
$$

(The last condition implies in particular that

$$
\begin{gathered}
h_{2}^{\prime}(\ell)=\frac{-E I h_{1}^{\prime \prime}(\ell)}{g_{h}} \\
\left.\theta_{2}(\ell)=\frac{-G J \theta_{1}^{\prime}(\ell)}{g_{\theta}}\right) \\
\mathcal{A}_{s} Y=\left|\begin{array}{c}
h_{2} \\
\frac{-E I h_{1}^{\prime \prime}(\ell)}{g_{h}} \\
\theta_{2} \\
\frac{-G J \theta_{1}^{\prime}(\ell)}{g_{\theta}} \\
E I h_{1}^{\prime \prime \prime}(\cdot) \\
-G J \theta_{1}^{\prime \prime}(\cdot)
\end{array}\right|
\end{gathered}
$$


The defined $\mathcal{A}_{s}$ is closed with dense domain, and compact resolvent. Moreover

$$
\begin{aligned}
\operatorname{Re}\left[\mathcal{A}_{s} Y, Y\right] & =\frac{-1}{g_{h}}(E I)^{2}\left|h_{1}^{\prime \prime}(\ell)\right|^{2}-\frac{1}{g_{\theta}}(G J)^{2}\left|\theta_{1}^{\prime}(\ell)\right|^{2} \\
& =-g_{h}\left|h_{1}^{\prime}(\ell)\right|^{2}-g_{\theta}\left|\theta_{1}(\ell)\right|^{2} .
\end{aligned}
$$

REMARK The last relation allows extension to the "limiting" cases:

$$
\begin{aligned}
& g_{h}=0 \quad \text { by adding the condition } h_{1}^{\prime \prime}(\ell)=0 \\
& g_{\theta}=0 \quad \text { by adding the condition } \theta_{1}^{\prime}(\ell)=0 .
\end{aligned}
$$

Next we define the linear operators $D$ and $K$ on $\mathcal{H}_{E}$ into $\mathcal{H}_{E}$ :

$$
Y=\left|\begin{array}{c}
h_{1} \\
h_{1}^{\prime}(\ell) \\
\theta_{1} \\
\theta_{1}(\ell) \\
h_{2} \\
\theta_{2}
\end{array}\right| ; \quad D Y=\left|\begin{array}{c}
0 \\
0 \\
0 \\
0 \\
M^{-1} D_{a} \mid h_{2} \\
\theta_{2}
\end{array}\right| ; \quad K Y=\left|\begin{array}{c}
0 \\
0 \\
0 \\
0 \\
M^{-1} K_{a} \mid h_{1} \\
\theta_{1}
\end{array}\right|
$$

Thus defined, $D$ and $K$ are bounded linear operators, their precise bounds being not of interest. Define

$$
\mathcal{A}=\mathcal{A}_{s}+U D+U^{2} K .
$$

Then $\mathcal{A}$ generates a $C_{0}$-semigroup; denote it $S(t), t \geq 0$. Also

$$
\operatorname{Re}[\mathcal{A} Y, Y]=\operatorname{Re}\left[\mathcal{A}_{s} Y, Y\right]+\pi \rho U^{2} \operatorname{Re}\left[\theta_{1}, \theta_{2}\right] .
$$

The semigroup $S(\cdot)$ is thus not necessarily a contraction for nonzero $U$. But the resolvent of $\mathcal{A}, R(\lambda, \mathcal{A})$, is compact and there are no eigenvalues in the half plane

$$
\operatorname{Re} \lambda>\sigma_{a}
$$

where $\sigma_{a}$ is the growth bound of the semigroup generated by $\mathcal{A}$, which of course depends on $U$.

Next for each $t \geq 0$, define the linear operators $L_{0}(t), L_{1}(t)$ on $\mathcal{H}_{E}$ into $\mathcal{H}_{E}$ by

$$
L_{0}(t) Y=\left|\begin{array}{c}
0 \\
0 \\
0 \\
0 \\
M^{-1}\left|\begin{array}{c}
\ell_{00}(t) V \\
\ell_{10}(t) V
\end{array}\right|
\end{array}\right|
$$




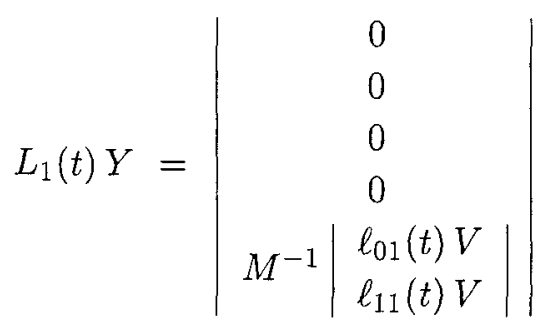

where

$$
V=\left|\begin{array}{l}
h_{1} \\
\theta_{1} \\
h_{2} \\
\theta_{2}
\end{array}\right| .
$$

Then our abstraction version (or time domain formulation) is the evolution-convolution equation in a Hilbert space:

$$
\dot{Y}(t)=\mathcal{A} Y(t)+\int_{0}^{t} L_{0}(t-\sigma) Y(\sigma) d \sigma+\int_{0}^{t} L_{1}(t-\sigma) \dot{Y}(\sigma) d \sigma
$$

where we shall need to impose some additional properties of the operators $L_{0}(\cdot), L_{1}(\cdot)$, such as

$$
\int_{0}^{\infty}\left\|L_{0}(t)\right\| e^{-\sigma t}+\int_{0}^{\infty}\left\|L_{1}(t)\right\| e^{-\sigma t}<0, \quad \sigma>0
$$

and

$L_{1}(t)$ coverges strongly to $L_{0}(\infty)$, linear bounded as $t \rightarrow \infty$

$$
L_{0}(t) \text { coverges strongly to } L_{1}(\infty) \text {, linear bounded as } t \rightarrow \infty \text {. }
$$

Equations like (42) have been discussed in the pure mathematics literature (e.g., [20]) but unfortunately are much too abstract to provide answers to the questions of interest to us here. Following [17] we start by taking Laplace Transforms in (42). Defining

$$
\hat{Y}(\lambda)=\int_{0}^{\infty} e^{-\lambda t} Y(t) d t, \quad \operatorname{Re} \lambda>\sigma_{a}
$$

we have

$$
(\lambda I-\mathcal{A}) \hat{Y}(\lambda)-\hat{L}_{0}(\lambda) \hat{Y}(\lambda)-\hat{L}_{1}(\lambda)(\lambda \hat{Y}(\lambda)-Y(0))=Y(0)
$$

or

$$
\left(\lambda I-\mathcal{A}-\hat{L}_{0}(\lambda)-\lambda \hat{L}_{1}(\lambda)\right) \hat{Y}(\lambda)=\left(I-\hat{L}_{1}(\lambda)\right) Y(0) .
$$


We shall refer to

$$
\left(\lambda I-\mathcal{A}-\hat{L}_{0}(\lambda)-\lambda \hat{L}_{1}(\lambda)\right) Y=Z
$$

as the generalized resolvent equation and

$$
\left(\lambda I-\mathcal{A}-\hat{L}_{0}(\lambda)-\lambda \hat{L}_{1}(\lambda)\right)^{-1}
$$

the generalized resolvent. We may then state: (cf [19]) without proof:

THEOREM 2 For any $\lambda$, either

$$
\left(\lambda I-\mathcal{A}-\hat{L}_{0}(\lambda)-\lambda \hat{L}_{1}(\lambda)\right) Y=0
$$

for $\|Y\| \neq 0$, or

$$
\left(\lambda I-\mathcal{A}-\hat{L}_{0}(\lambda)-\lambda \hat{L}_{1}(\lambda)\right)
$$

has a bounded inverse.

TheOREM 3 Call $\lambda$ such that

$$
\left(\lambda I-\mathcal{A}-\hat{L}_{0}(\lambda)-\lambda \hat{L}_{1}(\lambda)\right) Y=0, \quad\|Y\| \neq 0
$$

an "aeroelastic mode." The aeroelastic modes are countable in number for each fixed $M, U$ and only a finite number can have positive real part.

We can show that the aeroelastic modes are precisely the eigenvalues of the infinitesimal generator of a semigroup. We can also view this another way. We make a state space formuation of the linear system represented by the evolution-convolution equation (42). By state space representation we mean the representation

$$
\left.\begin{array}{rl}
\dot{Z}(t) & =\mathcal{A}_{c} Z(t)+\mathcal{B} u(t) \\
Y(t) & =\mathcal{C} z(t)
\end{array}\right\}
$$

where $\mathcal{A}_{c}$ is the infinitesimal generator of a $C_{0}$-semigroup, $\mathcal{B}$ is linear bounded and $\mathcal{C}$ is closed linear. In the present case $\mathcal{B}=0$, and the controls are included already in "feedback" form. The state space needs to be a Banach Space. Such a representation for the case $L_{0}(\cdot)=0$ is given in [21], and is readily generalized to the present case. We should note that the representation (46) allows us to check controllability and stabilizability for any given control scheme. $Y(\cdot)$ represents only the structure state and $Z(\cdot)$ includes a "stand in" for the aerodynamic state. 


\subsection{Calculation of Aeroelastic Modes: Flutter Speed}

From the practical point of view perhaps the most important problem is to track the aeroelastic modes as a function of $U$, for fixed $M$, in order to determine stability. For $\alpha=0$ this is carried out in [13] and the extension to nonzero $\alpha$ is straightforward.

Thus we need to start with "unwinding" (45), returning to the Laplace Transform version of (1). We show that the modes are the zeros of a function

$$
d(M, \lambda, U)
$$

which is analytic in $\lambda$ except for a logarithmic singularity for $\lambda \leq 0$. We need to define the roots as a single-valued function of $U$ - define the "root locus." For $U=0$ we obtain the structure modes - two sequences — the "bending" modes and the "pitching" modes. We begin with:

$$
\lambda_{k}(\lambda, M, 0)=i \omega_{k}
$$

the structure modes, and we show that

$$
\left.\frac{\partial}{\partial \lambda} d(M, \lambda, U)\right|_{\lambda=i \omega_{k}} \neq 0
$$

so that we can via the usual implicit function theory define the roots $\lambda_{k}(M, U)$ as a function of $U$ with

$$
-\frac{\frac{\partial d}{\partial U}}{\frac{\partial d}{\partial \lambda}}=\frac{\partial \lambda(M, U)}{\partial U} .
$$

See [13] for the details. If, for example,

$$
\lambda_{k}(M, 0)=i \omega_{k}
$$

is the the $k$ th bending mode we keep calling $\lambda_{k}(M, U)$ the root locus of the $k$ th bending mode. Let

$$
\sigma_{k}(M, U)=\operatorname{Re} \lambda_{k}(M, U) .
$$

Then

$$
\sigma_{k}(M, 0)=0 .
$$

The curve of $\sigma_{k}(M, U)$ is called the "stability curve." We show that

$$
\left.\frac{\partial \sigma_{K}(M, U)}{\partial U}\right|_{U=0}=\text { constant }\left(\frac{-1}{M}\right)
$$


for all $k$ with the constant depending on whether it is a pitching mode or a bending mode. This enables us to define flutter speed $U_{F}(M)$ as the first time

$$
\sigma_{k}(M, U)=0, \quad \frac{\partial \sigma_{k}(M, U)}{\partial U}>0 .
$$

We are also able [13] to deduce a good many properties of $U_{F}(M)$ as a function of $M$, but not nearly enough! Much work still remains to be done especially for $M$ close to 1 , being in particular not continuous at $M=1$.

As shown in [4] the theory can help explain the occurrence of the Transonic Dip due to nonzero angle of attack - and should also explain that due to camber observed in computations [22], [23]. The point is that even though the system is nonlinear the stability as we have seen is still determined by the linearized model.

The solution $Y_{k}$ of the modal equation corresponding to the $k$ th aeroelastic mode $\lambda_{k}(M, U)$ is called the "mode shape" and we can express the time-domain ("unsteady") solution of the aeroelastic convolutionevolution equation in terms of the elements in $Y_{k}$ even though it is not an eigenfunction expansion - see [17], [19]. This is not of much use in application to the flutter problem except to indicate the nature of the instability, since the aerodynamic initial conditions can never be determined. It is nevertheless of mathematical interest.

\section{References}

[1] Meyer, R.E., Introduction to Mathematical Fluid Mechanics. Dover Publications, 1982.

[2] Friedmann, P.P. "The Renaissance of Aeroelasticity and Its Future." Journal of Aircraft, Vol. 36, No. 1 (1999), pp. 105-121.

[3] Goland, M. "The Flutter of a Uniform Cantilever Wing," Journal of Applied Mechanics, ASME, Vol. 12, No. 4 (1954), pp. A197-A208.

[4] Balakrishnan, A.V. "On the Transonic Small Disturbance Potential Equation." Submitted to AIAA Journal.

[5] Nixon, D. "Basic Equations for Unsteady Transonic Flow." Chapter 2 in: Unsteady Transonic Aerodynamics. Progress in Astronautics and Aeronautics Series, Vol. 120. Edited by David Nixon. American Institute of Astronautics and Aeronautics, 1989. Pp. 57-73.

[6] Ferrari, C. and Tricomi, F.G. Transonic Aerodynamics. Translated by Raymond H. Cramer. Translation of Aerodinamica transonica. Academic Press, New York, 1968.

[7] Bers, L. Mathematical Aspects of Subsonic and Transonic Gas. Surveys in Applied Mathematics, Vol. 3. John Wiley and Sons, New York, 1958.

[8] Guderley, K.G. The Theory of Transonic Flow. Pergamon Press, 1962. 
[9] Balakrishnan, A.V. "On the (Non-numeric) Mathematical Foundations of Linear Aeroelasticity." In: Fourth International Conference on Nonlinear Problems in Aviation and Aerospace. Edited by Seenith Sivasundaram. European Conference Publications, 2003. Pp. 11-41.

[10] Cole, J.D. and Cook, L.P. Transonic Aerodynamics. North-Holland Series in Applied Mathematics and Mechanics, Vol. 30. Elsevier Science Pub. Co., 1986.

[11] Balakrishnan, A.V. "Possio Integral Equation of Aeroelasticity Theory." Journal of Aerospace Engineering, Vol. 16, No. 4 (2003).

[12] Possio, C. "L'azione aerodinamica sul profile oscillante in un fluido compressibile a velocità iposonora." L'Aerotecnica. Vol. 18, No. 4 (1938).

[13] Balakrishnan, A.V. and Iliff, K.W. "A Continuum Aeroelastic Model for Inviscid Subsonic Bending-Torsion Wing Flutter." In: Proceedings of International Forum on Aeroelasticity and Structural Dynamics, June 4-6, 2003, Amsterdam.

[14] Chorin, A.J. and Marsden, J.E. A Mathematical Introduction to Fluid Mechanics. 3rd Edition. Texts in Applied Mathematics, Vol. 4. Springer-Verlag, New York, 1993.

[15] Bisplinghoff, R.L., Ashley, H. and Halfman, R.L. Aeroelasticity. AddisonWesley Publishing Co., 1955.

[16] Fung, Y.C. An Introduction to the Theory of Aeroelasticity. Dover, New York, 1983.

[17] Balakrishnan, A.V. "Aeroelastic Control with Self-straining Actuators: Continuum Models." In: Smart Structures and Materials 1998: Mathematics and Control in Smart Structures. Edited by Vasundara V. Varadan. Proceedings of SPIE, Vol. 3323. Pp. 44-54.

[18] Balakrishnan, A.V. "Dynamics and Control of Articulated Anisotropic Timoshenko Beams." In: Dynamics and Controls of Distributed Systems. Edited by H.S. Tzou and L.A. Bergman. Cambridge University Press, United Kingdom, 1998. Pp. 121-201.

[19] Balakrishnan, A.V. "Subsonic Flutter Suppression Using Self-straining Actuators." Journal of the Franklin Institute, Vol. 338 (2001), pp. 149-170.

[20] Prüss, J. Evolutionary Integral Equations and Applications, Birkhauser, 1993.

[21] Balakrishnan, A.V. "Representing the Convolution-Semigroup Equation of Aeroelasticity as a Pure Semigroup Equation." Presented at ICNPAA 2000, Third International Conference on Nonlinear Problems in Aviation and Aerospace, Daytona Beach, Florida, May 2000. Unpublished report.

[22] Bendiksen, O.O. "Transonic Flutter." AIAA Paper No. 2002-1488. Presented at: 43rd AIAA/ASME/ASCE/AHS/ASC Structures, Structural Dynamics, and Materials Conference, Denver, Colorado, April 22-25, 2002.

[23] Schultz, S. "Transonic Aeroelastic Simulation of a Flexible Wing Section." Agard Report 822. March 1998. 